\title{
Active Model Based Carotid Ultrasonic Data Segmentation
}

\author{
Alexandre Moreau-Gaudry ${ }^{1}$, Philippe Cinquin ${ }^{1}$ and Jean-Philippe Baguet ${ }^{2}$ \\ 1 TIMC-IMAG \\ Institut Albert Bonniot, 38706 La Tronche cedex, France \\ \{Alexandre.Moreau-Gaudry,Philippe.Cinquin\}@imag.fr \\ 2 Department of Internal Medicine and Cardiology \\ Hôpital Michallon, CHU Grenoble, France
}

\begin{abstract}
To diagnose cardiovascular diseases, the most significant mortality causes in industrialized countries, the study of the carotid artery wall plays a preferential part. This paper presents a three dimensional active model based carotid ultrasonic data segmentation. A C1 continuous surface, defined by a deformable skeleton and envelope, includes the Y-shape topology and gray level as a priori knowledge. It is automatically fitted to a sequence of $2.5 \mathrm{D}$ ultrasonic branching data by an iterative quadratic method. The quality of the fit is illustrated by figures. Result of this 3D fitting can be used as initial position for quantification of the intima-media thickness.
\end{abstract}

\section{Introduction}

Among cardiovascular diseases, coronary diseases represent the most important part in terms of frequency, morbidity and mortality. Lesions of the carotid artery wall (infraclinic atherosclerosis) reflect their effects. With the introduction of 2.5D ultrasound imaging (2D ultrasonic data located in $3 \mathrm{D}$ space), 3D analysis of the whole artery appears to be feasible. To initialize this study, a global branching model fits ultrasonic slices in an automatic way. It is made possible by the use of a priori knowledge : the $\mathbf{Y}$-shape topology of the model and gray level modelling. This defines an absolute reference system. Tissular modifications (intima plus media thickness) and clinical parameters (degree of stenosis, plaque volume), evaluated from the result fitting, can be followed in this reference system, improving so the reproducibility.

\section{An Active Branching Model}

In [1], we mention the particularity of the branching topology and explain why we don't use deformable models or reconstruction methods like [7,4]. Recently, in [5], a branching reconstruction was performed from two dimensional carotid CT images. Contrary to these, ultrasonic images are often of poor quality. The 
maximum of a priori knowledge has to be used to compensate the lack of informations. A natural and powerful a priori knowledge is the particular Y-shape of the branching. This knowledge is introduced by the use of a global branching model. Although topologically adaptative deformable models quoted in [6] have been shown to be promising in segmenting vascular structures in $2 \mathrm{D}$ and $3 \mathrm{D}$ images, we develop in [1] an explicit C1 continuous surface based on a deformable skeleton and envelope (see figure 1). It is totally defined by 24 model parameters. Thanks to the add of gray level a priori knowledge, a rough fully automatic carotid ultrasonic data segmentation will be realized.

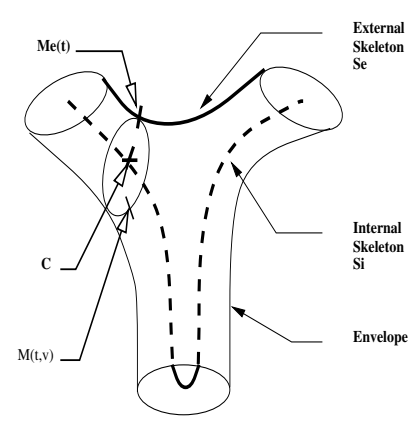

Fig. 1. Surface Description .
$S_{e}$, the external skeleton, represents the upper branching convexity. It is a planar curve parameterized by $t, t \in[-1 . .1]$. $S_{i}$, the internal skeleton, is the set of centers $\mathrm{C}$ of planar curves, continuously built on $S_{e}$. Each planar curve (superquadric [3]) are parameterized by $v, v \in[-\pi . . \pi]$. The envelope of $S_{i}$ defines the branching surface. By a one-to-one mapping between $S_{e}$ and $S_{i}$, an explicit equation $M(t, v)$ of the surface is obtained, which was a main point for the numerical efficiency. For complementary explanations, see [1].

\section{Data Collection}

Data are obtained with collaboration of the Department of Internal Medicine and Cardiology at the Grenoble University Hospital. Echographic images are acquired by a HP SONOS (Hewlett-Packard, Santa Clara, California, USA) ultrasonic system at a frequency of $50 \mathrm{~Hz}$. Figure 2 explains how to compute $2.5 \mathrm{D}$ data from these 2D data.

Because of the high frequency of acquisition, we are in front of an huge amount of information, which requires an automatic treatment. This will be possible thanks to the a priori knowledge contained in the branching shape. In a first approach, only cross-sectional data acquired during one cardiac cycle along the carotid artery are treated.

\section{Methods}

Let $X=\left(X_{j}, j=1 . .24\right)$ be the vector of 24 model parameters, $d X$, an elementary shifting in the space of model parameters; $M(t, v ; X)$, a point of current parameters $(\mathrm{t}, \mathrm{v})$ on the surface of parameter $X, d M(t, v ; X)$, the elementary movement of a point $M(t, v ; X)$ in the real $3 \mathrm{D}$ space. 


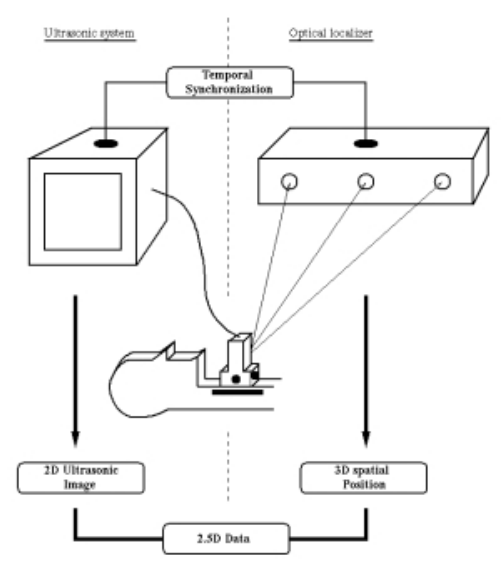

\section{Fig. 2. Acquisition of 2.5D ultrasonic data.}

2D images are acquired on a HP ultrasonic system at a frequency of $50 \mathrm{~Hz}$. During the medical exploration, the ultrasonic system is connected to an optical localizer (Optotrak Northern Digital Inc.).

To collect $2.5 \mathrm{D}$ data, a six degrees-offreedom optical tracking device attached to the ultrasound probe is used. The optical tracking system works with infrared diodes put on the probe which are then located by three fixed CCD cameras (Optotrak Northern Digital Inc.) at a frequency of $100 \mathrm{~Hz}$. Position and orientation of the echographic planes are thus obtained during all the exploration.

Thanks to a temporal synchronization between echographic and spatial data, 3D spatial coordinates for each $2 \mathrm{D}$ points of echographic plane can be computed.

\subsection{Initialization}

Shape initialization Thanks to the particular shape of the model, it is made easy. We choose manually four points on the $2.5 \mathrm{D}$ data, corresponding roughly to the top of the bifurcation position (the lowest point of the external skeleton) and the three branching extremities (the bottom, the right and the left extremities of the $\mathrm{Y})$. A initial set $X 0$ of parameter is then automatically computed from positions and distances between these different points. $X 0$ is chosen so that the initial branching surface is in the lumen of the vessel.

Gray level initialization From this initial position, the intersections of the surface (see 4.2) with the ultrasonic planes are computed (closed curves). For each point of the curves, a gray level profile (10 pixel) is extracted towards the inside. We model the inside by a gaussian of mean $\mu$ and of variance $\sigma$.

\subsection{Calculating a Suggested Movement}

Intersection computing To determine surface intersection with ultrasonic planes, we compute first their intersections with three plane curves $C i, i=1 . .3$ judiciously chosen on the surface (see Figure 3). Two half spaces are defined by each ultrasonic plane. By determining the change of sign of the signed distance function, the exact intersections of planes with $C i$ are calculated by a Brent's method ([9]). From these points or germs, we compute, by a similar process, step by step, points $M_{i}\left(t_{i}, v_{i} ; X\right)$ on the surface intersection, taking into account of the provenance direction. 


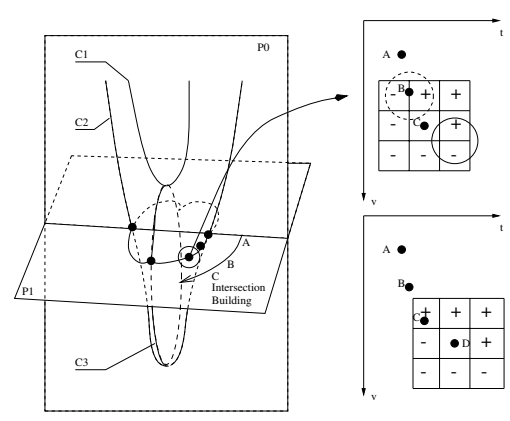

\section{Fig. 3. Intersection computing: an overview.}

Left: from germ A, intersection between the ultrasonic plane P1 and C2, we build step by step the intersection: points A,B,C.

Right: at the current point $\mathrm{C}$, the signed distance map in its neighborhood is computed. After having identified the two changes of signs (circle with solid and dashed line), the point which maximizes the sum of distances with the three last points A,B,C is chosen and computed. This makes the process to go forward by choosing D (circle with solid line). The process is then iterated.

Suggested movement $d M_{i}$ Let $\overrightarrow{n_{i}}$ be the projection in the current plane of the normal $\vec{N}_{i}$ of the surface in $M_{i}$. For each point $M_{i}$, the elementary movement $d M_{i}$ in the $2 \mathrm{D}$ plane along $\overrightarrow{n_{i}}$ according to the gaussian gray model is computed: shifting from the inside (-10 pixels) of the branching intersection to the outside $(+5$ pixels $)$, we go on until pixel intensity $I$ at current point along the profile does not verify gaussian threshold: $\left(\frac{I-\mu}{\sigma}\right)^{2}>1$. If $M_{i}+d M_{i}$ is outside, a weight $\alpha_{i}$ of 1 is associated to it. On the contrary, if $M_{i}+d M_{i}$ is inside, to retract the surface, a weight proportional to $\left\|d M_{i}\right\|^{2}$ is chosen. By this asymmetry of balancing, a controlled inflating strategy is created, which prevents to go through the border of the vessel. $d M_{i}$ computed, the movement along $\vec{N}_{i}$ is inferred from it.

\subsection{Suggested Shifting $d X$}

Computing $d X$ from $n$ elementary movements $d M_{i}$, weighted by $\alpha_{i}, i=1 . . n$, was a main point to establish a relation between the space parameter and the real 3D space. Each point $M_{i}\left(t_{i}, v_{i} ; X\right) \equiv M_{i}(X)$ is seen as a function from $\Re^{24}$ to $\Re^{3}$ : it associates to the vector $\mathrm{X}$, the $3 \mathrm{D}$ point $M_{i}(X)$ on the surface of parameter $X$. With an approximation at the first order, the following relation is achieved: $d M_{i}(X)=J_{M_{i}}\left(t_{i}, v_{i} ; X\right)(d X)$, where $J_{M_{i}}(X) \equiv J_{M_{i}}\left(t_{i}, v_{i} ; X\right)$ is the jacobian matrix of function $M_{i}$. Because we have an explicit definition of the surface, an explicit expression of $J_{M_{i}}(X)$ is computed with Maple software (Maple V Release 4). To determine the shifting $d X$ from $d M_{i}, i=1 . . n$, we choose to minimize in $\Re^{24}$ the quadratic problem Q (1), which comes down to resolve a linear system in $\Re^{24}$.

$$
Q=\sum_{i=1}^{n} \alpha_{i}\left\|d M_{i}\left(t_{i}, v_{i} ; X\right)-J_{M_{i}}\left(t_{i}, v_{i} ; X\right) d X\right\|^{2}
$$




\subsection{Algorithm}

The natural process consists then to iterate the last three steps. To stop the process, we define a cost function, sum of $2 \mathrm{D}$ elementary movements in each plane along the outline: each movement is counted positive when it goes outside the surface, and negative in the other case. Process is stopped when the cost function is minimal.

\subsection{Modelling a Gray Level Appearance According to the Incidence of Ultrasonic Rays}

Because ultrasonic images are often of poor quality, to model the relation between the incidence of ultrasonic rays and the gray levels in the image is a precious a priori knowledge: we have indeed to remember that the wall of the vessel is particularly well identifiable if ultrasonic rays are normal to its surface, and vice versa.

Let $\vec{d}$ be the incidence direction of ultrasounds. At first approximation, the border of the vessel is circular on cross-sections. A polar angle $\theta$ of nil value in the direction $\vec{d}$ (see figure 4 ) is then naturally introduced. This angle is the parameter which gives us informations on the expected quality of gray levels. A new a priori knowledge is injected under the form of a statistical gray level modelling coming from [10], and function of $\theta$. This model is applied on the result of the precedent step, to determine in an more acute way the limit of the vessel: we indeed have to be close to the vessel wall to compute a realistic angle with the incidence direction of ultrasounds.
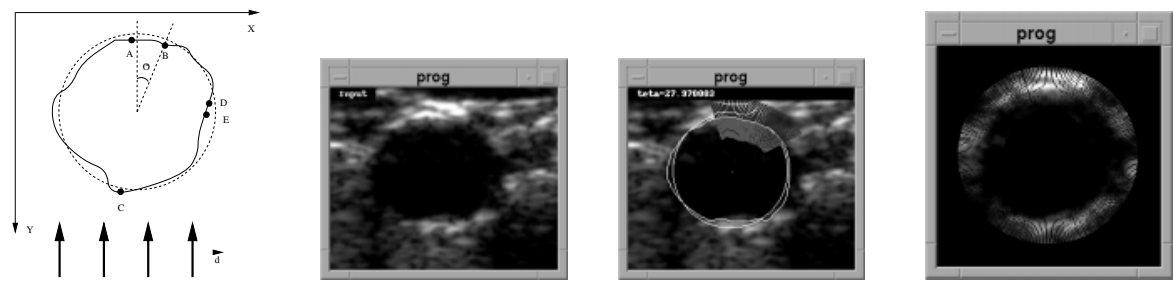

Fig. 4. A parameter which gives informations on the expected quality of gray levels : $\theta$.

Left: $\vec{d}$ is the incidence direction of ultrasonic rays. For $\theta$ values close to zero or $\pi$, (points $A, B, C$ ), the ultrasonic echo is of good quality and this part of the image is meaningful. For $\theta$ values close to $\frac{p i}{2},-\frac{p i}{2}$, (points $D, E$ ), we have a lack of information.

Middle left: Example of image on which gray level extraction will be applied.

Middle right: Extraction in course; the best circle fitting the border of the vessel, manually determined, can be seen.

Right: Result of the gray level profile extraction in each point of the border. 
Model Building The statistical model is built from $m$ ultrasonic images of the vessel $(m=6)$. Each image $i m_{j}, j=1 . . m$ is manually segmented. To extract gray level profiles according to the incidence angle of the ultrasonic rays, we fit, according to [13], the best circle on the border. A scaling transform is applied on each image to align circles with respect to each other, so that the images represent the same informations. Using a tri-linear interpolation, for each image $i m_{j}$, for each degree $\theta, \theta=1 . .360$, a gray level profile $p_{j, \theta}$ of 20 pixel centered on the border is extracted (see figure 4). Following [10,8], a Principal Components Analysis on gray level profiles of identical angle $p_{j, \theta=c s t}, j=1 . . m$ is performed, in order to recover the principal components of the wall: we thus obtain, for each $\theta$, the mean $m_{\theta}$, and keep the eigenvectors $p_{\theta, k}$ corresponding to 95 percent of gray level information (four or five eigenvectors: $k=1 . .4,5$ ). Thus, 360 gray level profiles along the border of the vessel are defined.

Model fitting Let $M_{i}$ be a current point of the vessel wall. To fit the gray level model with informations contained in the image in $M_{i}$ to calculate a more accurate displacement, we compute the angle $\theta$ between $\vec{d}$ and $\overrightarrow{n_{i}}$, the previously defined direction of profile extraction. 20 gray level profiles along $\overrightarrow{n_{i}}$ are then extracted. For each profile, its Mahalanobis distance ([7]) from the mean $m_{\theta}$, which is a measure of how well it fits the model, is computed. The point, for which this distance is minimum, is the new $M_{i}+d M_{i}$.

\section{Results}

After a manual initialization, the model modifies its shape by an active process to fit the border of the vessel. Figure 5 shows different intersections of the surface with ultrasonic planes. One can clearly notice a visual improvement with regard to the border of the vessel, particularly in the critical region of the bifurcation (plane 21). Furthermore, it appears that the model is not enough inflated. Improvements concerning the stop measure or the displacement of points have to be done.

\section{Discussion and Conclusion}

\subsection{Advantages and Pitfalls}

Advantages Because ultrasonic images are renowned for their poor quality, a priori knowledge is required to deal with them. In our case, the intrinsic information carried by our model is its shape. It makes possible an easy 3D and gray model initialization, which makes feasible the autonomous active search of the border of the vessel. A priori shape modelling is an active research topic ([10]). Only few methods are able to automatically describe the division of one artery in two branches [4,2]: to our knowledge, no explicit continuous active deformable branching model incorporating the $\boldsymbol{Y}$-shape topology as an a priori information had been developed. Furthermore, the compactness of the branching description, totally defined by 24 parameters, has to be underlined. The relatively 

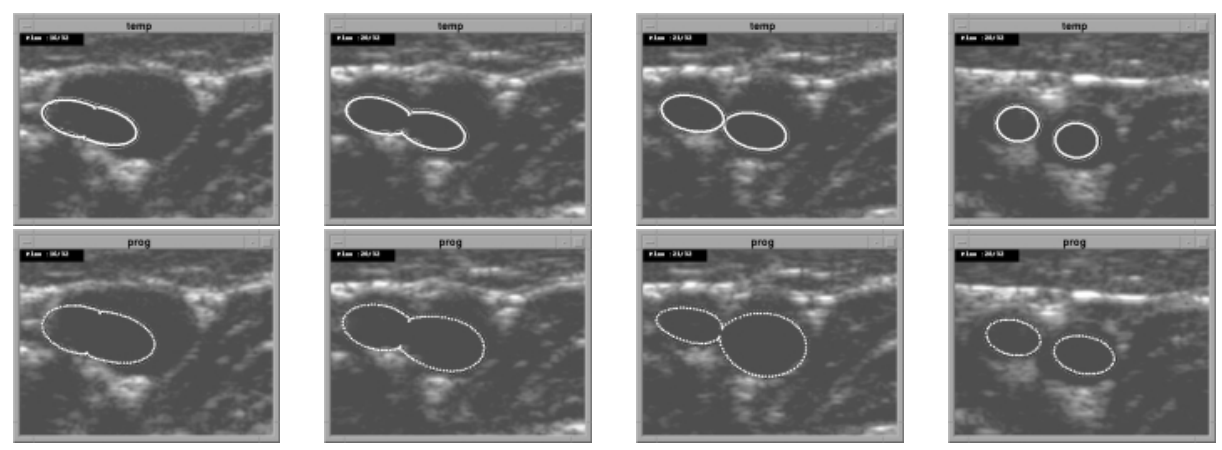

Fig. 5. Result.

Line 1: Intersection of the initial surface with ultrasonic planes.

Line 2: Intersection of the final surface with the same ultrasonic planes as for line 1 .

small number of parameters results in a certain stiffness of deformation. This is the cost to pay for compactness. It would be possible to improve it by the use of deformable superquadrics with local and global deformations [11], or by other methods [12]. We don't adopt this strategy principally for two reasons: the implementation is heavy, and the result that we obtain is sufficient for the next step of the vessel study (quantification of the thickness of the wall). Our result provides a mean position of the carotid during the cardiac cycle, position from which we're going to start to study more precisely the wall of the vessel in other slices, at different time of the cardiac cycle.

necessary to evaluate the impact of the initial position for the model fitting, especially with regard to the spatial initialization (near or away from the bifurcation). The fitting is relatively slow on a HP A 9000/715 workstation. The calcul of intersections of the surface with ultrasonic planes represents the blocking step. A C (and not $\mathrm{C}++$ ) or a parallel implementation could be certainly more efficient.

\subsection{Perspectives}

Given this initial position, the addition of the statistical gray level a priori information (build but not totally tested) will be useful for a more acute wall study. The addition of ultrasonic acquisitions of the same vessel under different incidences will be interesting to rebuilt more precisely the vessel: the next natural step will be the introduction of longitudinal slices. Our geometric model is the first step to an accurate quantification (quantification of the variations of the wall thickness during a cardiac cycle). It will be a measurement tool to evaluate new medical therapeutics tested to slow down atherosclerosis evolution. It could be an element in medical decision making, in order to optimize the cost/benefit equation. 
Our data collection corresponds to a cross sectional study. We need some longitudinal information to test the reproducibility and the parameters sensibility of our model. This tool is promising. It could be used for all the branching vessels acquired by ultrasonic system (femoral, renal, popliteal divisions). It is known that ultrasonic slices are very noisy. Investigations by CT scan or MRN could be interesting. The use of our model could be extended, particularly to cerebral, coronary or hepatic branching vessels.

At last, in physiological medical research, our model which creates a virtual branching surface from 3D real data could be included in hemodynamic vascular model to test the local repercussion of turbulence on the atherosclerosis outcome.

\section{Acknowledgments}

This work was supported by Hewlett-Packard and Medasys Digital Systems.

\section{References}

1. A. Moreau-Gaudry, J.P. Baguet, and P. CINQUIN A New Branching Model: Application to Carotid Ultrasonic Data. Medical Image Computing and ComputerAssisted Intervention-MICCAI'98-LNCS, pages 1049-1056, 1998.

2. D.Attali E.Ferley, M.P.Cani-Gascuel. Skeletal Reconstruction of Branching Shapes. The Eurographics Association, 1997.

3. F.Solina and R.Bajcsy. Recovery of parametric models from range images:The case for superquadrics with global deformations. IEEE Transactions on Pattern Analysis and Machine Intelligence, 12(2):131-147, February 1990.

4. V.Juhan B.Nazarian K.Malkani R.Bulot J.M.Bartoli and J.Sequeira. Geometrical modeling of abdominal aortic aneurysms. CVRED-MRCAS, pages 243-252, 1996.

5. G.Abdoulaev, S.Cadeddu, G.Delussu, M.Dinizelli, L.Formaggia, A.Giachetti, E.Gobbetti, A.Leone, C.Manzi, A.Scheinine, M.Tuveri, A.Varone, A.Veneziani, G.Zanetti and A.Zorcolo. ViVa: The Virtual Vascular Project. IEEE Transactions on Infromation Technology in Biomedecine, 2:268-273, December 1998.

6. T. McInerney and D. Terzopoulos. Deformable models in medical image analysis: a survey. Medical Image Analysis, 1(2):91-108, 1996.

7. S. Benayoun C.Nastar N.Ayache. Dense non-rigid motion estimation in sequence of 3d images using differential constraints. CVRMed, 1995.

8. C. Nastar B. Moghaddam A. Pentland. Generalized matching for recognition and retrieval in an image database. Proceedings of the Third International Conference: Communicating by Image and Multimedia, May 1996.

9. W.H.Press W.T.Vetterling S.A.Teukolsky and B.P.Flannery. Numerical recipies in c:Second edition.

10. T.F.Cootes A.Hill C.J.Taylor and J.Haslam. The use of active shape models for locating structures in medical images. Image and Vision Computing, 12(6):355366, July 1994.

11. D. Terzopoulos and D. Metaxas. Dynamic 3D models with local and global deformations: Deformable superquadrics. IEEE Transactions on Pattern Analysis and Machine Intelligence, 13(7):703-714, July 1991.

12. D. Terzopoulos and M. Vasilescu. Sampling and reconstruction with adaptive meshes. In IEEE Computer Society Conference on Computer Vision and Pattern Recognition (CVPR'91), pages 70-75, Maui, Hawaii, June 1991.

13. A.Bjorck. Numerical Methods for Least Squares Problems. SIAM, 1996. 